\title{
Nguồn thông tin, lòng tin và sự lựa chọn nơi mua rau: Nghiên cứu ở thành phố Hồ Chí Minh
}

\section{Information, trust and market outlet choice for vegetables: The case study in Ho Chi Minh City}

\author{
Lê Thị Tuyết Thanh ${ }^{1 *}$ \\ ${ }^{1}$ Trường Cao đẳng Công Thương Thành phố Hồ Chí Minh, Việt Nam \\ "Tác giả liên hệ, Email: tuyetthanh293@ gmail.com
}

THÔNG TIN

DOI: $10.46223 /$ HCMCOUJS. econ.vi.13.1.1616.2018

Ngày nhận: 15/12/2017

Ngày nhận lại: 16/02/2018

Duyệt đăng: 14/03/2018

Tù khóa:

an toàn thực phẩm, lựa chọn nơi mua, rau

\section{TÓM TẮT}

Sau một thời gian dài không thay đổi trong thói quen chọn nơi mua rau hay thực phẩm nói chung, người tiêu dùng Thành phố Hồ Chí Minh (TP.HCM) thời gian gần đây đã bắt đầu dịch chuyển mạnh mẽ từ các kênh truyền thống sang các kênh hiện đại như siêu thị hay siêu thị mini. Một trong các nguyên nhân thay đổi là vấn đề thực phẩm bẩn mà cụ thể là rau tại các kênh phân phối truyền thống không đảm bảo an toàn vệ sinh thực phẩm. Nghiên cứu này phân tích tác động của (1) lòng tin đối với người bán và quản lý nhà nước về VSATTP và (2) kênh tìm kiếm thông tin về VSATTP đến tần suất mua rau tại các kênh bán lẻ. Số liệu tự điều tra từ 254 người mua rau tại TP.HCM được phân tích bằng mô hình Seemingly Unrelated Negative Binomial. Kết quả nghiên cứu cho thấy những người tin ở chính sách quản lý VSATTP có xu hướng chọn mua rau ở chợ nhiều hơn và do đó cải thiện chính sách quản lý an toàn thực phẩm đối với rau sẽ giúp cải thiện hoạt động của chợ truyền thống. Ngoài ra những người tìm hiểu thông tin qua truyền thanh truyền hình và báo chí có tần suất mua rau ở siêu thị cao. Cửa hàng RAT có thể nhờ kênh truyền miệng để tăng lượng khách hàng vì những người tìm hiểu thông tin qua kênh truyền miệng có xu hướng mua rau ở cửa hàng RAT nhiều hơn.

\begin{abstract}
Being dominated by the traditional markets after a long time, the retail market for vegetables has recently experienced a dramatic change, as Ho Chi Minh City consumers start moving toward modern store formats such as supermarkets and minisupermarkets. One of the reasons is that the traditional retail channels fail to assure food safety. This study analyzes the
\end{abstract}


Keywords:

choice of purchase location, food safety, vegetables

impacts of (1) trust in sellers and governance concerning food safety and (2) channels of information related to food safety, on the frequency of purchasing vegetables at different retail channels. Data collected from a survey of 254 vegetable purchasers in Ho Chi Minh City was analyzed using the Seemingly Unrelated Negative Binomial Regression. The results indicate that those who trust food safety governance tend to purchase vegetables more frequently at traditional markets, and thus enhancing food safety governance would improve the performance of traditional markets. In addition, those who seek information related to food safety from radio and television tend to purchase vegetables more frequently at supermarkets. Finally, purchasers are driven to vegetable specialty stores by word of mouth and therefore these stores may attract more purchasers via this information channel.

\section{Giới thiệu}

Rau là thành phần chính, quan trọng trong mỗi bữa ăn của người Việt (Hoang \& Nakayasu, 2006; Wertheim-Heck, 2015), là nguồn cung cấp vitamin, khoáng chất và các chất dinh dưỡng đặc biệt khác (Cheng et al., 2016; Huang, Pan, Wu, Han, \& Chen, 2014). Việc tiêu dùng rau hằng ngày có khả năng ngăn ngừa các nguy cơ từ ung thư, bệnh tim mạch và béo phì (Cheng et al., 2016). Ý thức về việc tiêu dùng rau vì sức khỏe ngày càng được nâng cao đã khiến lượng tiêu thụ rau trên toàn thế giới tăng lên nhanh chóng trong 10 năm qua. Báo cáo kết quả điều tra thị hiếu người tiêu dùng TP.HCM do Trung tâm tư vấn và hỗ trợ doanh nghiệp Sở Nông nghiệp và Phát triển Nông thôn thực hiện vào năm 2016 cho thấy mức tiêu thụ của người tiêu dùng đối với rau, củ trung bình khoảng 400 gram/người/ngày, trong đó rau ăn lá chiếm $63,5 \%$, rau củ quả chiếm $36,5 \%$.

Trong suốt một thập kỷ từ 2000 - 2010, thói quen chọn nơi mua rau của người tiêu dùng Thành phố Hồ Chí Minh hầu như không thay đổi với chợ truyền thống là kênh mua chủ đạo với $90 \%$ người mua (VEBIMO, 2010). Tuy nhiên những năm gần đây đã có sự thay đổi lớn. Năm 2016, theo kết quả khảo sát của Sở NN\&PTNN (2016) người tiêu dùng đa phần mua rau tại các siêu thị, siêu thị mini $(53,4 \%)$ và chợ lẻ $(44,8 \%)$, còn lại một số ít là tại các chợ đầu mối. Trong đó, chủ yếu người tiêu dùng mua rau an toàn (RAT) tại các siêu thị $(77,18 \%)$, một số rất ít tại các chợ lẻ $(20,81 \%)$ và chợ đầu mối $(2,01 \%)$. Nhu cầu của người tiêu dùng ngày càng thay đổi theo hướng an toàn hóa.

Chính vì mối quan tâm rất lớn đến tính an toàn nên khi người tiêu dùng quyết định mua rau họ thường thu thập thông tin từ nhiều nguồn khác nhau. Theo kết quả từ Trung tâm tư vấn và hỗ trợ doanh nghiệp (Sở NN\&PTNN, 2016), nguồn thông tin mà họ thu thập chủ yếu đến từ báo, đài $(54,05 \%)$, poster, giới thiệu tại nơi bán $(21,62 \%)$, internet $(16,76 \%)$, bạn bè đồng nghiệp $(7,57 \%)$. Vì vậy mà các kênh thông tin có thể tác động rất lớn đến sự lựa chọn nơi mua rau. 
Ở Việt Nam đã có một số nghiên cứu về sự lựa chọn nơi mua rau hay thực phẩm nói chung. Maruyama và Trung (2007) và Mergenthaler, Weinberger, và Qaim (2009) phân tích tác động của các yếu tố đến sự lựa chọn nơi mua bằng các mô hình probit riêng biệt. Phân tích bằng mô hình probit đòi hỏi phải định nghĩa biến lựa chọn theo kiểu có hay không mua tại một kênh nào đó trong một đơn vị thời gian. Cách này không thật sự phản ánh được mức độ mua nhiều hay ít tại mỗi kênh. Chưa kể việc ước lượng các mô hình probit riêng lẻ cho mỗi kênh là không hợp lý vì sự lựa chọn các kênh nhìn chung có tương quan với nhau và vì vậy cần ước lượng các phương trình một cách đồng thời. Ngoài ra, mô hình nhị phân dạng này không phân tích được tần suất mua ở các kênh bán lẻ.

Lapar và cộng sự (2009) thì sử dụng mô hình multinomial logit. Mô hình này đòi hỏi phải phân loại người tiêu dùng vào một kênh bán lẻ cụ thể vì trong mô hình này các phương án lựa chọn (các kênh bán lẻ) đòi hỏi phải loại trừ lẫn nhau. Điều này có thể không chính xác vì người mua có thể mua ở nhiều kênh và đôi khi tần suất mua ở các kênh không khác nhau đáng kể.

Nghiên cứu này phân tích các yếu tố ảnh hưởng đến tần suất mua rau tại các kênh bán lẻ của người tiêu dùng Thành phố Hồ Chí Minh sử dụng mô hình Seemingly Unrelated Negative Binomial (SUNB). Mô hình này cho phép ước lượng hệ phương trình tần suất mua rau tại các kênh một cách đồng thời, theo nghĩa nó cho phép tần suất mua tại các kênh có tương quan với nhau và do đó khắc phục được các nhược điểm của các nghiên cứu trước ở Việt Nam. Số liệu thu thập từ khảo sát 254 người tiêu dùng tại Thành phố Hồ Chí Minh. Trong các yếu tố ảnh hưởng, nghiên cứu này đặc biệt quan tâm đến yếu tố lòng tin đối với người bán và quản lý vệ sinh an toàn thực phẩm (VSATTP) của chính phủ, cũng như các kênh thông tin mà người tiêu dùng tìm hiểu về VSATTP.

Nghiên cứu này phân loại các kênh bán lẻ ra thành bốn nhóm: chợ truyền thống, siêu thị, cửa hàng chuyên kinh doanh rau an toàn (RAT) và các kênh khác. Chợ và siêu thị đại diện cho hai kênh truyền thống và hiện đại. Cửa hàng RAT cũng làm một hình thức bán lẻ hiện đại, nhưng được tách riêng ra vì nó nhấn mạnh đến tính an toàn của rau.

\section{Các nghiên cứu về sự lựa chọn nơi mua rau}

\subsection{Chợ truyền thống và siêu thị}

Có khá nhiều nghiên cứu phân tích sự lựa chọn nơi mua thực phẩm giữa hai kênh siêu thị và chợ truyền thống sử dụng mô hình Logit hay Probit như: Goldman và Hino (2005), Iton (2015), Maruyama và Wu (2014), Maruyama, Wu, và Huang (2016).

\subsection{Phân tích sụ lựa chọn một kênh cụ thể}

Một số nghiên cứu phân tích sự lựa chọn một kênh mua cụ thể nào đó so với nhóm kênh còn lại như: Palma, Emerson, và House (2003), Neven, Reardon, Chege, và Wang (2006), Zepeda và Li (2006), Zepeda (2009), Maples, Morgan, Interis, và Harri (2013), Suel \& cộng sự (2015). Bond, Thilmany, và Bond (2009) cũng quan tâm sự lựa chọn mua trực tiếp từ người sản xuất nhưng định nghĩa biến lựa chọn hơi khác. Nghiên cứu này phân tích các yếu tố tác động đến tần suất mua trực tiếp từ người sản xuất: (1) không bao giờ; (2) luôn luôn; (3) thỉnh thoảng. 
Mô hình sử dụng MNL, nhưng lẽ ra tác giả có thể dùng ordered probit model để cho đơn giản hơn.

\subsection{Phân tích sụ lụa chọn giữa nhiều kênh phân phối khác nhau}

Có khá nhiều nghiên cứu phân tích sự lựa chọn giữa nhiều kênh phân phối như: Staus (2011), Okello, Lagerkvist, Hess, Ngigi, và Karanja (2011), Dong và Stewart (2012), Kyureghian và Nayga (2013), Melis, Campo, Breugelmans, và Lamey (2015), Gido, Ayuya, Owuor, và Bokelmann (2016), Slamet và Nakayasu (2016).

\subsection{Mô hìh Multivariate Probit/Logit}

Biến phụ thuộc của các mô hình logit/probit và MNL/MNP đòi hỏi các phương án lựa chọn là loại trừ lẫn nhau và do vậy không cho phép trường hợp người mua lựa chọn nhiều kênh khác nhau cùng lúc. Tuy nhiên trên thực tế người mua có thể mua cùng lúc ở nhiều kênh và phân loại những người này vào một kênh cụ thể là không chính xác.

Có hai cách giải quyết. Một là quan sát trong một thời gian nhất định và xác định nơi mua thường xuyên nhất và coi đó là lựa chọn của người tiêu dùng. Cách này có nhược điểm là bỏ qua việc nhiều người tiêu dùng có thể mua ở các kênh khác, đặc biệt là khi các kênh khác này cũng có tần suất mua không thấp hơn đáng kể so với kênh được lựa chọn. Cách thứ hai là quan sát từng lần mua, nghĩa là mỗi lần mua là một quan sát và một cá nhân hay hộ gia đình có thể có nhiều quan sát trong khoảng thời gian nghiên cứu. Cách này giúp phân tích đầy đủ hơn, nhưng đòi hỏi thu thập số liệu chi tiết hơn. Hầu hết các nghiên cứu áp dụng cách thứ nhất. Ch có một số ít nghiên cứu quan sát từng lần mua thực phẩm, gồm: Staus (2011), Dong và Stewart (2012), Kyureghian và Nayga (2013), và Melis và cộng sự (2015).

Khác với logit/probit và multinomial logit, mô hình multivariate probit ước lượng một hệ phương trình đồng thời, mỗi phương trình cho một kênh mua sắm. Do vậy mô hình này cho

phép trường hợp người tiêu dùng lựa chọn nhiều hơn một nơi mua sắm. Điều này phù hợp thực tế hơn vì một người trong một giai đoạn thời gian có thể mua sắm ở nhiều nơi cùng lúc. Tuy vậy mô hình này phức tạp hơn và khó tính toán tác động biên nên ít nghiên cứu sử dụng. Một số nghiên cứu hiếm hoi áp dụng mô hình này là Verhoef, Neslin, và Vroomen (2007), Bai, Wahl, và McCluskey (2008).

Gorton, Sauer, và Supatpongkul (2011) sử dụng mô hình Bivariate Tobit. Mô hình này ước lượng hai phương trình đồng thời, giải thích tỷ trọng chi tiêu mỗi nhóm hàng tại chợ trời và siêu thị. Đây có thể coi là một dạng mở rộng của Bivariate Probit/Logit.

\subsection{Các phưong pháp khác}

Neven và cộng sự (2006), Moore (2006) và Meng, Florkowski, Sarpong, Chinnan, và Resurreccion (2014) sử dụng mô hình ordered logit. Mặc dù ordered logit là phù hợp với biến phụ thuộc có thứ bậc, việc phân tích tần suất mua sắm tại các kênh bằng các mô hình riêng rẽ như Meng và cộng sự (2014) là không phù hợp vì các quyết định này có mối quan hệ phụ thuộc lẫn nhau, phải sử dụng mô hình multivariate ordered logit/probit như Gorton và cộng sự (2011). 
Gorton và cộng sự (2011) phân tích các yếu tố tác động đến tần suất mua thực phẩm bằng mô hình ordered bivariate probit. Mô hình bivariate cho phép phân tích tần suất mua sắm tại hai kênh, đồng thời cho phép hai hành vi này tương quan lẫn nhau.

\subsection{Các biến giải thích}

Tuổi: Các nghiên cứu sử dụng tuổi của người phụ nữ đứng đầu gia đình (Dong \& Stewart, 2012). Một số nghiên cứu cho thấy tuổi không có tác động đến sự lựa chọn kênh mua rau hay thực phẩm (Iton, 2015; Maruyama và Wu, 2014; Slamet \& Nakayasu, 2016; Zepeda, 2009; Zepeda \& Li, 2006). Tuy nhiên nhiều nghiên cứu khác lại cho thấy sở thích chọn nơi mua rau khác nhau theo độ tuổi: Okello và cộng sự (2011), Staus (2011), Maruyama và cộng sự (2016), và Gido và cộng sự (2016).

Trình độ học vấn: Các nghiên cứu của: Goldman và Hino (2005), Zepeda và Li (2006), Zepeda (2009), Maples và cộng sự (2013), Kyureghian và Nayga (2013) cho thấy người đi chợ có trình độ cao hơn có xác suất lựa chọn siêu thị (hoặc các cửa hàng hiện đại) cao hơn. Trong khi đó Okello và cộng sự (2011), Gido và cộng sự (2016) lại thấy không có sự khác biệt trong các trình độ học vấn đến quyết định lựa chọn kênh mua rau.

Quy mô gia đình được khá nhiều nghiên cứu sử dụng như: Okello và cộng sự (2011), Dong và Stewart (2012), Maples và cộng sự (2013), Iton (2015), Slamet và Nakayasu (2016) và Gido và cộng sự (2016) nhưng ít khi có tác động đến quyết định chọn nơi mua rau và thực phẩm.

Thu nhập và chi tiêu: Một số nghiên cứu sử dụng thu nhập, chi tiêu, chi tiêu bình quân đầu người, hoặc tỷ lệ thu nhập chi cho thực phẩm. Một vài nghiên cứu nhận thấy thu nhập hoặc chi tiêu không có ảnh hưởng đến quyết định lựa chọn giữa các kênh như: Goldman và Hino (2005), Zepeda (2009) và Gido và cộng sự (2016).

Trong khi đó: Okello và cộng sự (2011), Dong và Stewart (2012), Maruyama và Wu (2014), Iton (2015), và Maruyama và cộng sự (2016) cho thấy thu nhập hoặc chi tiêu có tác động đến quyết định lựa chọn nơi mua của người tiêu dùng.

\subsection{Nhận thức, lòng tin và nguồn thông tin}

Maples và cộng sự (2013) dùng số câu trả lời đúng đối với những kiến thức liên quan đến nông nghiệp để đo lường nhận thức. Lòng tin về chất lượng của rau ở các cửa hàng dẫn đến xu hướng mua rau ở siêu thị và cửa hàng chuyên doanh thay vì chợ trời và lề đường (Okello et al., 2011). Nhận thức về rủi ro đối với sức khỏe của rau không an toàn cũng có tác động tương tự.

Thái độ đối với các khía cạnh khác nhau của thực phẩm và việc mua sắm cũng được phân tích. Phần lớn các biến về thái độ được đo lường theo đánh giá chủ quan bằng thang đo Likert.

Bond và cộng sự (2009) dùng khá nhiều biến số lạ ví dụ kênh tiếp nhận thông tin về thực phẩm và dinh dưỡng. 


\section{Phương pháp nghiên cứu}

Mô hình Logit/Probit hay MNL đòi hỏi hộ gia đình phải lựa chọn một kênh mua sắm duy nhất. Điều này không đúng với thực tế. Mô hình Multivariate Probit/Logit cho phép một hộ chọn nhiều kênh, nhưng cách này cũng chưa phản ánh được tần suất mua sắm. Ví dụ theo cách này, một hộ mua ở chợ 10 lần và ở siêu thị 1 lần trong tuần qua có thể được xem là mua ở cả hai kênh. Nhưng rõ ràng tần suất mua sắm ở hai kênh là khác xa nhau.

Do vậy nghiên cứu này đề xuất sử dụng mô hình Negative Binomial (NB - một dạng mở rộng của mô hình Poisson) để phân tích tần suất mua sắm tại các kênh khác nhau. Các kênh được phân tích gồm (1) chợ truyền thống, (2) siêu thị, (3) cửa hàng RAT và (4) các kênh khác. Biến phụ thuộc là số lần đi mua rau mỗi tuần tại từng kênh. Các biến số này có giá trị nguyên không âm, do vậy mô hình NB là phù hợp.

\subsection{Mô hình Seemingly Unrelated Negative Biomial (SUNB)}

Ở mô hình NB, xác suất để hộ $i$ mua rau $k$ lần ở kênh $j$ là

$$
P_{r}\left(y_{i j}=k\right)=\frac{e^{-\alpha \lambda}(\alpha \lambda)^{k}}{k !} \quad \lambda>0
$$

Số lần mua rau $y_{i j}$ lúc này có giá trị kỳ vọng: $E\left[y_{i j}\right]=\lambda$ và phương sai $\lambda+\alpha \lambda^{2}$. Ta có thể mô hình hóa số lần mua kỳ vọng này là một hàm của các biến $X$

$$
\lambda=e^{X_{i} \beta}
$$

Với $X_{i}$ là đặc điểm của hộ $i$. Các hệ số $\beta$ lúc này phản ánh tác động của đặc điểm hộ gia đình $X_{i}$ đến số lần mua sắm kỳ vọng $\lambda$. Đây chính là mô hình NB. Lưu ý: khi $\alpha=0$ thì phương sai của biến phụ thuộc trở thành $\lambda$ và do vậy bằng với giá trị kỳ vọng. Lúc này mô hình $\mathrm{NB}$ được đơn giản hóa thành mô hình Poisson.

Các hệ số $\beta$ của mô hình NB được ước lượng bằng phương pháp Maximum Likelihood với hàm log-likelihood

$$
\log L=\sum_{i=1}^{N}\left(-e^{X_{i} \beta}+y_{i j} X_{i} \beta-\log y_{i j} !\right)
$$

Mô hình NB, tuy vậy chỉ có thể phân tích tác động của các biến $X_{i}$ đến tần suất mua sắm tại một kênh cụ thể. Nếu phân tích tác động đến tần suất mua sắm của nhiều kênh khác nhau thì phải ước lượng một mô hình NB riêng biệt cho mỗi kênh. Tuy nhiên điều này đòi hỏi phải giả định rằng tần suất mua sắm tại các kênh là độc lập với nhau. Giả định này không hợp lý trên thực tế vì các kênh có tính thay thế lẫn nhau, cho nên một người đã mua ở kênh này sẽ có xu hướng ít mua ở kênh khác hơn.

Mô hình SUNB do Winkelmann (2000) xây dựng có thể giải quyết được vấn đề này. Nó cho phép ước lượng các phương trình NB cho các kênh mua sắm một cách đồng thời.

\subsection{Các biến giải thích}

Dựa vào các tác giả trước, nghiên cứu này sử dụng các đặc điểm của hộ gia đình và của người ra quyết định mua rau làm biến giải thích. 
Các biến giải thích chính của bài viết bao gồm lòng tin và các kênh thông tin. Đối với lòng tin, hai biến giả được sử dụng. Một để ch người mua rau có tin người bán khi họ giới thiệu rau họ bán là rau sạch hay không. Biến thứ hai để ch người mua có cho rằng quản lý nhà nước về vệ sinh an toàn thực phẩm là yếu kém hay không.

\section{Bảng 1}

Định nghĩa các biến số

\begin{tabular}{|c|c|}
\hline \multicolumn{2}{|r|}{ Định nghĩa, đơn vị đo lường } \\
\hline \multicolumn{2}{|c|}{ ĐẠC ĐIỀM NHÂN KHẦU CỦA CÁ NHÂN VÀ Hộ GIA ĐÌNH } \\
\hline Tuổi & Tuổi của người ra quyết định mua rau (năm) \\
\hline Thu nhập & $\begin{array}{l}\text { Thu nhập bình quân đầu người của hộ (triệu } \\
\text { đồng/người/tháng) }\end{array}$ \\
\hline Quy mô hộ & Số thành viên trong hộ (người) \\
\hline Trình độ học vấn & 1 = PTTH (nhóm tham chiếu) \\
\hline (các biến giả sẽ được đưa vào mô & 2 = Trung cấp \\
\hline hình hồi quy) & $3=$ Cao đẳng \\
\hline & 4 = Đại học \\
\hline & 5 = Sau Đại học \\
\hline Nghề nghiệp & 1 = Quản lý (nhóm tham chiếu) \\
\hline (các biến giả sẽ được đưa vào mô & 2 = Nhân viên văn phòng \\
\hline hình hồi quy) & 3 = Công nhân \\
\hline & 4 = Nội trợ \\
\hline & LÒNG TIN \\
\hline Lòng tin đối với người bán & $\begin{array}{l}\text { Biến giả: } 1=\text { Tin tưởng khi người bán nói rau họ } \\
\text { bán là RAT }\end{array}$ \\
\hline Lòng tin đối với quản lý nhà nước & $\begin{array}{l}\text { Biến giả: } 1 \text { = Cho rằng quản lý nhà nước về } \\
\text { VSATTP là yếu kém }\end{array}$ \\
\hline \multicolumn{2}{|c|}{ CÁC KÊNH TÌM HIỂU THÔNG TIN VỀ VSATTP } \\
\hline Truyền thanh truyền hình & Biến giả: 1 = Có \\
\hline Internet & Biến giả: 1 = Có \\
\hline Báo chí & Biến giả: 1 = Có \\
\hline Truyền miệng & Biến giả: 1 = Có \\
\hline
\end{tabular}

Nguồn: Kết quả phân tích dữ liệu của nhóm nghiên cứu

Các kênh tìm kiếm thông tin bao gồm truyền thanh và truyền hình, internet, báo chí và truyền miệng. Bốn biến giả được sử dụng để ch người mua có tìm kiếm thông tin qua từng kênh hay không. Bảng 1 trình bày định nghĩa chi tiết các biến được sử dụng trong mô hình SUNB.

Như đã đề cập, nghiên cứu này tập trung vào yếu tố lòng tin và các nguồn thông tin đến sự lựa chọn nơi mua rau. Cụ thể, nghiên cứu này kiểm định các giả thuyết sau: 
H1: lòng tin đối với quản lý nhà nuớc làm tăng tần suất mua rau ở chợ truyền thống, trong khi làm giảm tần suất mua rau ở các kênh hiện đại (siêu thị và cửa hàng rau an toàn). Điều này không có đề cập trong các kết quả nghiên cứu trước, nhung nhu đã trình bày, nguời Việt Nam thích mua ở chợ truyền thống và khi họ tin rằng các chính sách quản lý VSATTP là tốt thì họ sẽ yên tâm mua ở chợ truyền thống hơn

H2: việc tiếp cận các nguồn thông tin (cả 4 nguồn) sẽ làm giảm tần suất mua rau ở chợ truyền thống và làm tăng tần suất mua ở các kênh hiện đại. Lý do là một khi người mua có đầy đủ thông tin hơn, họ sẽ thấy rằng rau ở chợ truyền thống không được kiểm soát chặt

Riêng lòng tin đối với người bán không rõ tác động, vì người bán có thể là người bán ở cả 4 kênh bán lẻ.

\subsection{Dũ liệu và phương pháp thu thập}

Số liệu được thu thập bằng bảng câu hỏi điều tra người tiêu dùng rau ở Thành phố Hồ Chí Minh với kỹ thuật chọn mẫu theo định mức (Quota Sampling) với đặc tính kiểm soát là khu vực sinh sống. Đối tượng được phỏng vấn là người đảm nhận việc mua thực phẩm (đặc biệt là rau) một cách thường xuyên cho gia đình với số mẫu chia đều cho hai quận và hai huyện: Quận 3, Bình Thạnh, Nhà Bè và Củ Chi. Mỗi quận huyện phỏng vấn 70 người. Khảo sát được tiến hàng từ tháng $8 / 2016$ đến 11/2016. Tổng số phiếu phát ra là 280 phiếu. Sau khi kiểm tra lỗi logic, làm sạch dữ liệu, tổng số phiếu cuối cùng hợp lệ là 254 . Số liệu thu thập được gồm 72 phiếu ở Quận 3, 53 phiếu ở Bình Thạnh, 54 phiếu Nhà Bè và 75 phiếu ở Củ Chi.

\section{Kết luận phân tích}

\subsection{Mô tả mẫu khảo sát}

Thống kê mô tả mẫu quan sát được trình bày ở Bảng 2 . Trung bình mỗi tuần một người mua rau ở chợ truyền thống 4,8 lần; siêu thị 1,7 lần; trong khi số lần mua rau ở cửa hàng RAT và các kênh khác là không đáng kể. Tuy vậy, số lần đi mua cũng dao động đáng kể, với số lần mua tối đa ở chợ là 14 và ở các kênh khác là 3 . Sự dao động này cho phép hồi quy mô hình SUNB.

Độ tuổi trung bình của người được phỏng vấn là 29 , dao động từ 16 đến 60 . Thu nhập bình quân là 2,6 triệu đồng/người/tháng. Quy mô hộ bình quân là, 9 người, gần bằng với quy mô bình quân từ các cuộc khảo sát lớn.

Hình 1 trình bày tần suất mua rau trung bình của các nhóm trình độ học vấn khác nhau. Nhóm PTTH hoặc thấp hơn gồm 59 người, trung bình mua rau ở chợ 5 lần/tuần, siêu thị 1 lần/tuần, và cửa hàng RAT và các kênh khác là khoảng 0,1 đến 0,2 lần/tuần. Các thanh đứng biểu thị độ lệch chuẩn của biến số. Nhìn chung các nhóm học vấn có số hộ không quá khác biệt (59, 46, 71 và 64 cho các bậc từ Đại học trở xuống). Riêng nhóm Sau đại học ít hơn, chỉ có 14 người. 


\section{Bảng 2}

Thống kê mô tả

\begin{tabular}{|c|c|c|c|c|c|c|}
\hline Biến số & & $\begin{array}{l}\text { Số quan } \\
\text { sát }\end{array}$ & $\begin{array}{l}\text { Trung } \\
\text { bình }\end{array}$ & $\begin{array}{l}\text { Độ lệ̣ch } \\
\text { chuẩn }\end{array}$ & $\begin{array}{l}\text { Tối } \\
\text { thiểu }\end{array}$ & $\begin{array}{l}\text { Tối } \\
\text { đa }\end{array}$ \\
\hline \multicolumn{7}{|c|}{ Số lần mua rau tại... (lần/tuần) } \\
\hline - Chọ & & 254 & 4.760 & 2.406 & 0 & 14 \\
\hline - Siêu thị & & 254 & 1.664 & 2.009 & 0 & 9 \\
\hline - Cửa hàng RAT & & 254 & 0.189 & 0.691 & 0 & 7 \\
\hline Khác & & 254 & 0.220 & 0.595 & 0 & 3 \\
\hline \multicolumn{7}{|c|}{ ĐẶC ĐIỂM HỘ GIA ĐİNH } \\
\hline Tuổi (năm) & & $254^{\circ}$ & 29.114 & 8.066 & 16 & 60 \\
\hline $\begin{array}{l}\text { Thu nhập bình quân } \\
\text { đồng/năm) }\end{array}$ & (triệu & 254 & 2.586 & 1.381 & 0.6 & 12 \\
\hline Quy mô hộ (người) & & 254 & 3.933 & 1.345 & 1 & 10 \\
\hline
\end{tabular}

Nguồn: Kết quả phân tích dữ liệu của nhóm nghiên cứu

Quan sát đồ thị cho thấy người có trình độ cao hơn có xu hướng mua ở chợ ít hơn và mua ở siêu thị nhiều hơn. Trong khi đó, số lần mua tại cửa hàng RAT và các kênh khác không có sự dao động đáng kể theo trình độ học vấn.

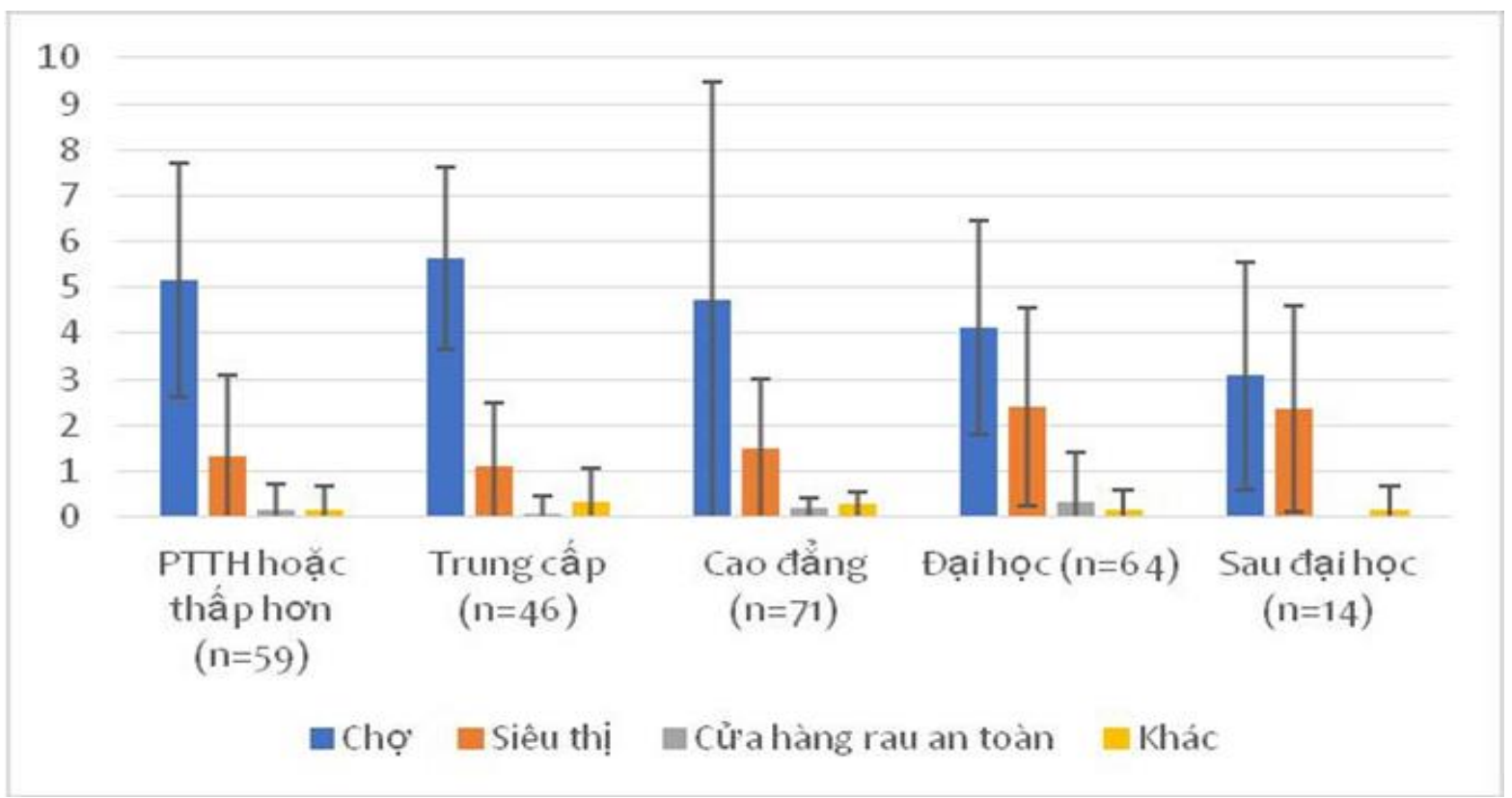

Hình 1. Tần suất mua rau tại các kênh phân theo trình độ học vấn

Tương tự, Hình 2 trình bày tần suất mua rau tại các kênh phân theo nhóm nghề nghiệp. Nhóm quản lý có 97 người, trung bình mua rau ở chợ 4,5 lần, siêu thị 1,6 lần và hai kênh còn lại khoảng 0,2 đến 0,3 lần. Nhìn chung có sự khác biệt trong tần suất mua rau tại các kênh giữa các nhóm nghề nghiệp. Nhóm nhân viên văn phòng và công nhân mua rau ở chợ nhiều lần hơn hai nhóm còn lại, trong khi nhóm công nhân ít mua rau ở siêu thị hơn so với các nhóm khác. Nhóm công nhân cũng có xu hướng mua ở các kênh khác nhiều hơn các nhóm còn lại. 


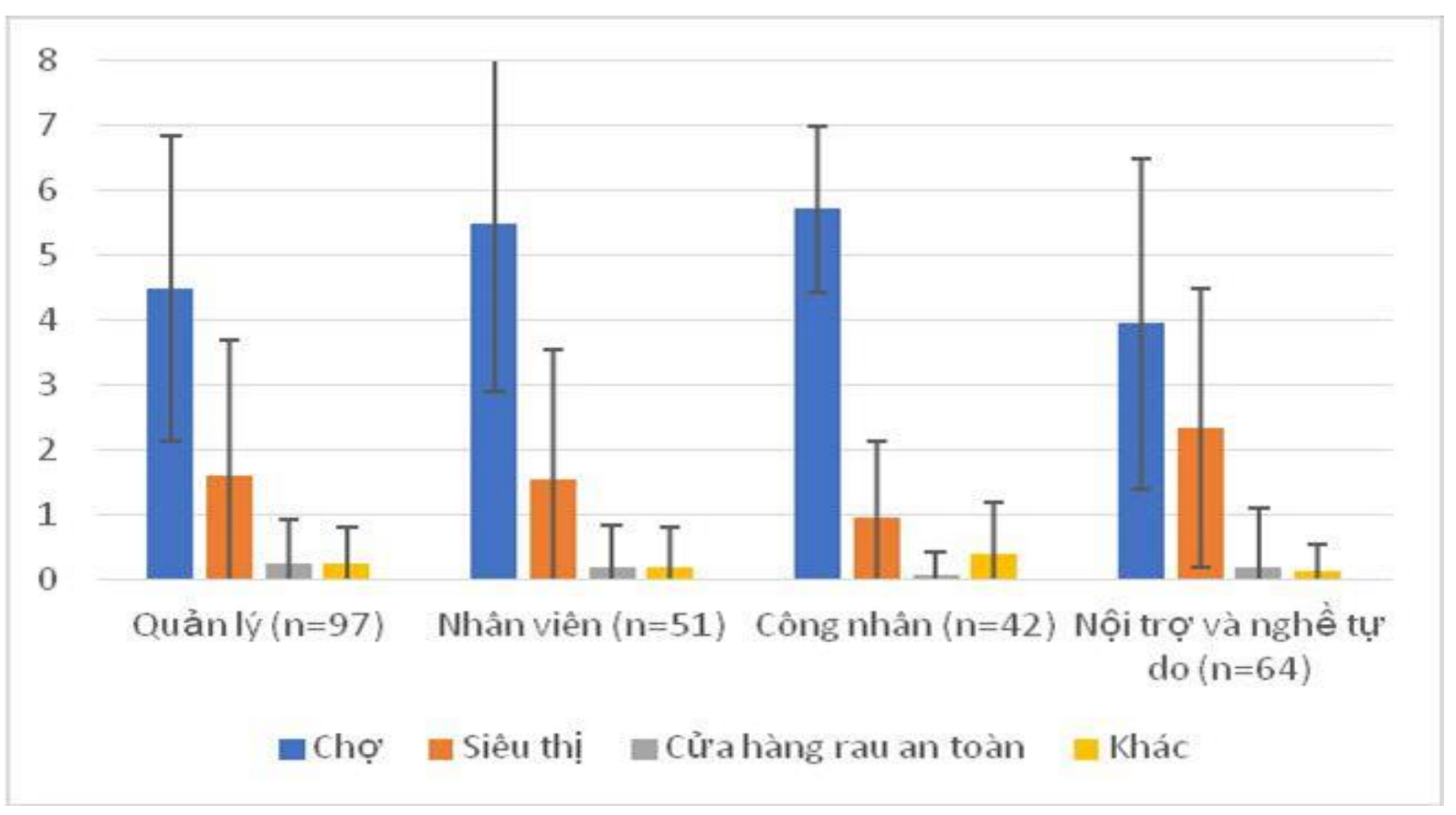

Hình 2. Tần suất mua rau tại các kênh phân theo nghề nghiệp

Hình 3 trình bày tần suất mua rau phân chia theo các nhóm tin tưởng người bán và tin tưởng vào quản lý nhà nước về VSATTP. Tần suất mua rau tại các kênh phân theo nhóm tin tưởng người bán được trình bày ở Hình $\mathrm{a}$. Nhìn chung người tin tưởng người bán có xu hướng mua ở siêu thị nhiều hơn và mua ở chợ ít hơn. Tần suất mua ở cửa hàng và các kênh khác lại không có khác biệt.

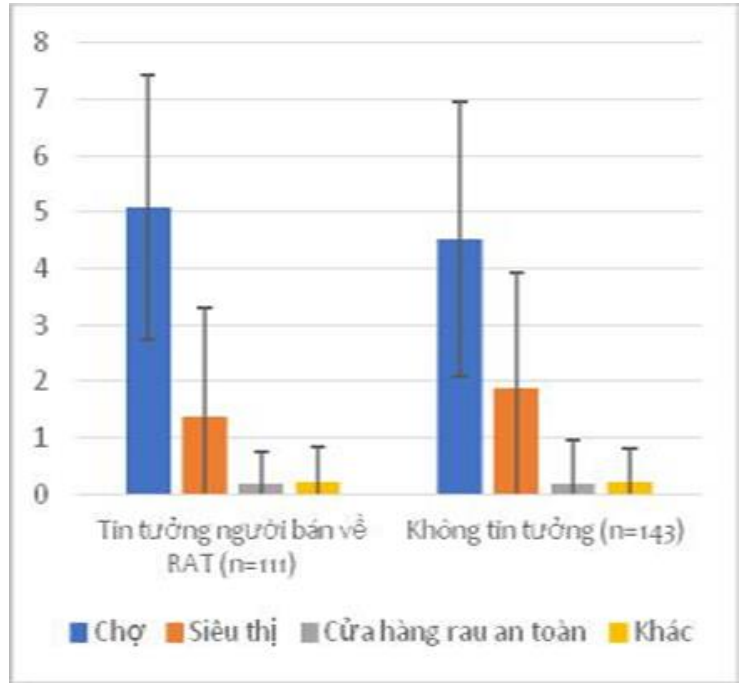

(a) Tần suất mua rau phân theo lòng tin đối với người bán

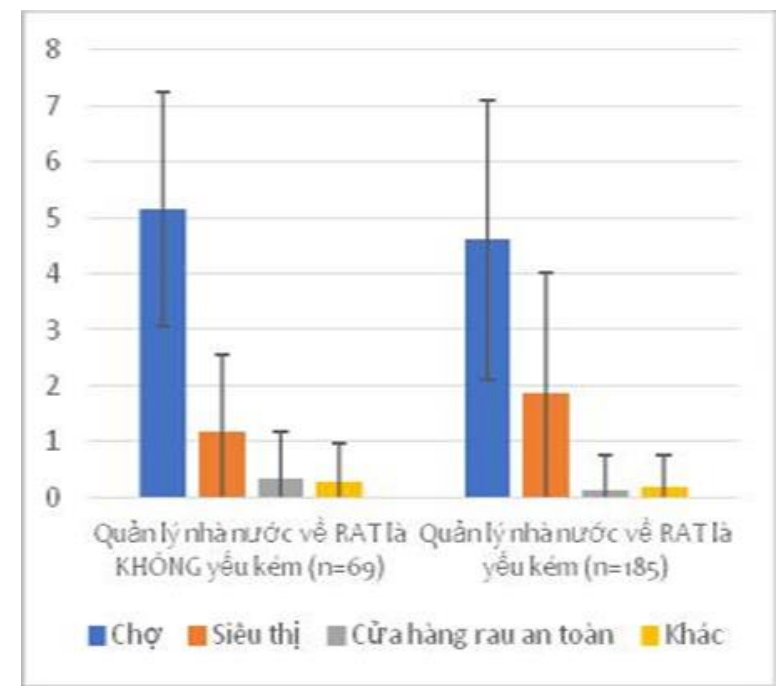

(b) Tần suất mua rau phân theo lòng tin đối với quản lý nhà nước

Hình 3. Lòng tin và tần suất mua rau tại các kênh 
Hình $3 a$ trình bày tần suất mua rau tại các kênh của hai nhóm tin tưởng và không tin tưởng quản lý nhà nước về VSATTP. Nhóm tin tưởng có xu hướng mua ở chợ nhiều hơn và mua ở siêu thị ít hơn. Nói chung khi lòng tin đối với người bán hay với chính phủ tăng lên thì người mua có xu hướng mua ở chợ truyền thống nhiều hơn.

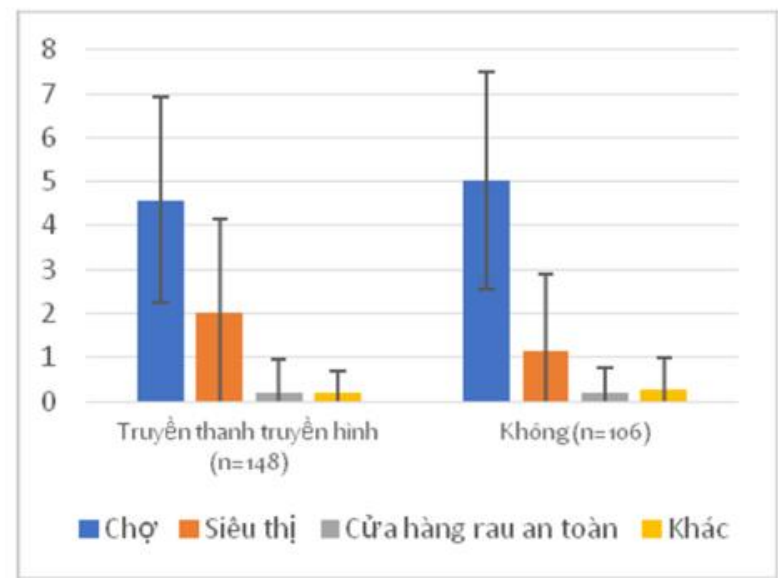

(a) Truyền thanh truyền hình

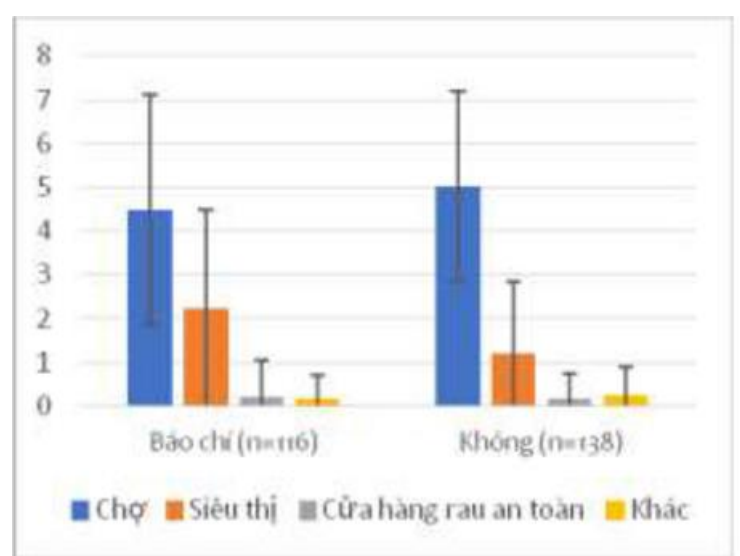

(c) Báo chí

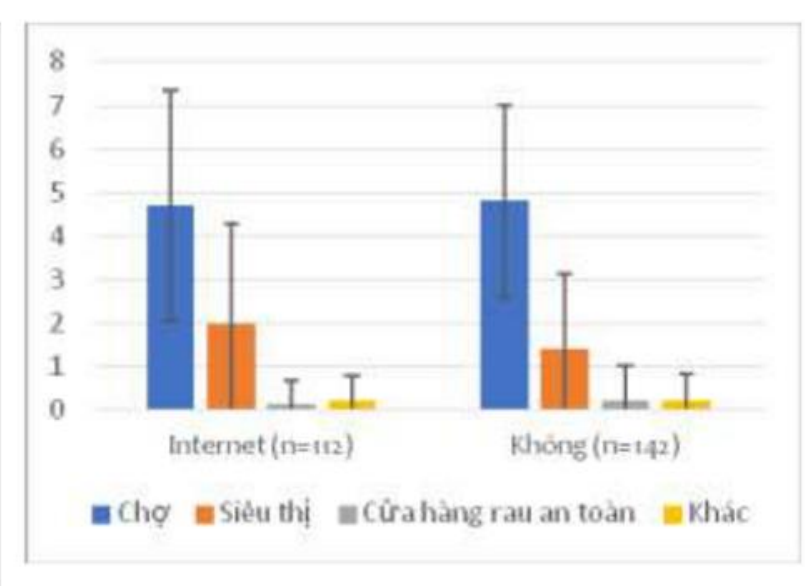

(b) Internet

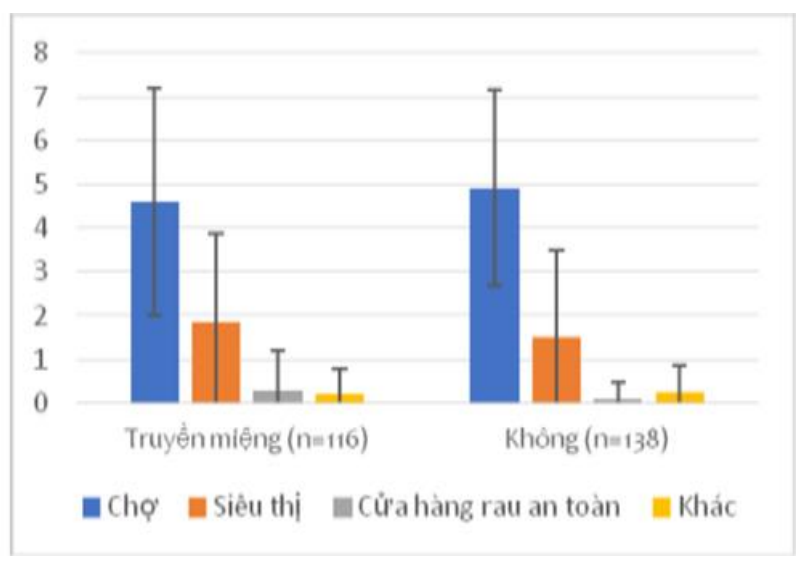

(d) Truyền miệng

Hình 4. Nguồn thông tin về VSATTP và tần suất mua rau tại các kênh

Hình 4 so sánh tần suất mua rau tại các kênh giữa các nhóm có và không có tìm hiểu thông tin từ các kênh truyền thanh truyền hình, internet, báo chí và truyền miệng. Nhìn chung, nhóm có tìm hiểu thông tin từ các nguồn đều có xu hướng mua ở chợ ít hơn và mua ở siêu thị nhiều hơn so với nhóm không có tìm hiểu thông tin. Tuy nhiên có thể nhận thấy rằng tác động của các kênh thông tin là khác nhau. Nhóm tìm hiểu thông tin qua truyền thanh truyền hình và báo chí có số lần mua rau ở siêu thị cao gấp đôi so với nhóm không tìm hiểu thông tin qua các kênh này. Trong khi đó, tác động của kênh truyền miệng là không đáng kể. 


\subsection{Kết quả hồi quy}

Bảng 3 trình bày kết quả hồi quy mô hình SUNB cho bốn kênh mua rau. Bốn phương trình cho bốn kênh được ước lượng đồng thời, với biến phụ thuộc là tần suất mua rau tương ứng tại mỗi kênh. Như đã đề cập, mô hình SUNB cho phép tần suất mua rau tại các kênh có tương quan với nhau và do đó hệ số ước lượng không bị chệch như khi ước lượng riêng lẻ.

Kết quả hồi quy cho thấy các đặc điểm nhân khẩu có tác động khác nhau đến tần suất mua rau tại các kênh. Tuổi nhìn chung không có tác động đến tần suất mua rau tại các kênh, ngoại trừ chợ truyền thống. Cụ thể là người cao tuổi hơn ít mua rau ở chợ hơn. Thu nhập ch có tác động đến tần suất mua rau tại cửa hàng RAT và các kênh khác, không có tác động đến tần suất mua rau tại chợ truyền thống và siêu thị. Nhìn chung người có thu nhập cao hơn có xu hướng ít mua ở chợ truyền thống hơn, chuyển dần sang mua ở các kênh khác, tuy nhiên ch có mức tăng ở cửa hàng RAT và các kênh khác là thay đổi đáng kể. Quy mô hộ gia đình cũng có tác động tương tự như thu nhập. Hộ có nhiều thành viên hơn có xu hướng mua ở cửa hàng RAT và các kênh khác nhiều hơn.

\section{Bảng 3}

Kết quả hồi quy mô hình SUNB (N = 254)

\begin{tabular}{|c|c|c|c|c|}
\hline & Chọ’ & Siêu thị & Cửa hàng RAT & Khác \\
\hline Tuổi (năm) & $\begin{array}{c}-0.011 * * \\
(0.005)\end{array}$ & $\begin{array}{c}0.016 \\
(0.011)\end{array}$ & $\begin{array}{c}0.006 \\
(0.031)\end{array}$ & $\begin{array}{l}-0.026 \\
(0.024)\end{array}$ \\
\hline $\begin{array}{l}\text { Thu nhập bình quân đầu } \\
\text { người } \\
\text { (triệu đồng/tháng) }\end{array}$ & $\begin{array}{l}-0.052 \\
(0.036)\end{array}$ & $\begin{array}{c}0.086 \\
(0.076)\end{array}$ & $\begin{array}{l}0.373 * \\
(0.192)\end{array}$ & $\begin{array}{l}0.277 * * \\
(0.133)\end{array}$ \\
\hline $\begin{array}{l}\text { Quy mô hộ } \\
\text { (người) }\end{array}$ & $\begin{array}{c}-0.03 \\
(0.024)\end{array}$ & $\begin{array}{c}0.037 \\
(0.061)\end{array}$ & $\begin{array}{c}0.313 * * \\
(0.133)\end{array}$ & $\begin{array}{l}0.253 * * \\
(0.117)\end{array}$ \\
\hline Trình độ học vấn & & & & \\
\hline PTTH trở xuống & \multicolumn{4}{|c|}{ Nhóm tham chiếu } \\
\hline Trung cấp & $\begin{array}{l}0.0003 \\
(0.087)\end{array}$ & $\begin{array}{l}-0.062 \\
(0.248)\end{array}$ & $\begin{array}{c}0.39 \\
(0.718)\end{array}$ & $\begin{array}{l}0.861 * \\
(0.501)\end{array}$ \\
\hline C円 & $\begin{array}{l}-0.118 \\
(0.116)\end{array}$ & $\begin{array}{c}0.106 \\
(0.294)\end{array}$ & $\begin{array}{l}1.424 * * \\
(0.7)\end{array}$ & $\begin{array}{c}0.955 \\
(0.618)\end{array}$ \\
\hline ĐH & $\begin{array}{l}-0.107 \\
(0.137)\end{array}$ & $\begin{array}{c}0.449 \\
(0.306)\end{array}$ & $\begin{array}{l}1.648^{* *} \\
(0.682)\end{array}$ & $\begin{array}{c}0.336 \\
(0.675)\end{array}$ \\
\hline Sau ĐH & $\begin{array}{l}-0.424^{*} \\
(0.247)\end{array}$ & $\begin{array}{c}0.35 \\
(0.417)\end{array}$ & $\begin{array}{c}-13.31 * * * \\
(0.919)\end{array}$ & $\begin{array}{c}0.246 \\
(1.061)\end{array}$ \\
\hline Nghề nghiệp & & & & \\
\hline Quản lý & \multicolumn{4}{|c|}{ Nhóm tham chiếu } \\
\hline Nhân viên văn phòng & $\begin{array}{c}0.241 * * \\
(0.103)\end{array}$ & $\begin{array}{c}0.046 \\
(0.255)\end{array}$ & $\begin{array}{l}-0.279 \\
(0.639)\end{array}$ & $\begin{array}{l}0.296 \\
(0.625)\end{array}$ \\
\hline Công nhân & $\begin{array}{l}0.237 * * * \\
(0.087)\end{array}$ & $\begin{array}{l}-0.373 \\
(0.231)\end{array}$ & $\begin{array}{l}-1.212^{*} \\
(0.667)\end{array}$ & $\begin{array}{c}0.738 \\
(0.505)\end{array}$ \\
\hline Nội trọ̆ & $\begin{array}{c}0.082 \\
(0.104)\end{array}$ & $\begin{array}{l}-0.11 \\
(0.202)\end{array}$ & $\begin{array}{l}-0.507 \\
(0.756)\end{array}$ & $\begin{array}{l}-0.511 \\
(0.493)\end{array}$ \\
\hline \multicolumn{5}{|c|}{ Lòng tin } \\
\hline Tin tưởng người bán về RAT & $\begin{array}{c}-0.131 * * \\
(0.065)\end{array}$ & $\begin{array}{c}0.274^{*} \\
(0.16)\end{array}$ & $\begin{array}{l}-0.567 \\
(0.412)\end{array}$ & $\begin{array}{c}0.337 \\
(0.323)\end{array}$ \\
\hline
\end{tabular}




\begin{tabular}{|c|c|c|c|c|}
\hline & Chọ & Siêu thị & Cửa hàng RAT & Khác \\
\hline Cho rằng quản lý $\mathrm{NN}$ về $\mathrm{RAT}$ & $-0.126^{*}$ & $0.458 * * *$ & $-1.374 * * *$ & -0.36 \\
\hline là yếu kém & $(0.065)$ & $(0.169)$ & $(0.399)$ & $(0.369)$ \\
\hline \multicolumn{5}{|c|}{ Các kênh tìm hiểu thông tin về VSATTP } \\
\hline $\begin{array}{l}\text { Truyền thanh truyền hình } \\
(\text { Có = 1) }\end{array}$ & $\begin{array}{l}-0.103 \\
(0.063)\end{array}$ & $\begin{array}{c}0.444 * * * \\
(0.165)\end{array}$ & $\begin{array}{l}-0.115 \\
(0.462)\end{array}$ & $\begin{array}{l}-0.407 \\
(0.332)\end{array}$ \\
\hline $\begin{array}{l}\text { Internet } \\
(\text { Có = 1) }\end{array}$ & $\begin{array}{c}0.072 \\
(0.063)\end{array}$ & $\begin{array}{l}-0.018 \\
(0.167)\end{array}$ & $\begin{array}{l}-0.895^{*} \\
(0.485)\end{array}$ & $\begin{array}{l}-0.246 \\
(0.339)\end{array}$ \\
\hline Báo chí & -0.027 & $0.43 * *$ & 0.319 & -0.372 \\
\hline$\left(C_{0}^{\prime}=1\right)$ & $(0.067)$ & $(0.17)$ & $(0.394)$ & $(0.321)$ \\
\hline Truyền miệng & $-0.135 * *$ & $\begin{array}{c}0.216 \\
(0.159)\end{array}$ & $\begin{array}{c}1.308 * * * \\
(0.431)\end{array}$ & $\begin{array}{l}-0.161 \\
(0.352)\end{array}$ \\
\hline Constant & $\begin{array}{c}2.367 * * * \\
(0.231)\end{array}$ & $\begin{array}{c}-1.575 * * * \\
(0.494)\end{array}$ & $\begin{array}{c}-4.236^{* * *} \\
(1.469)\end{array}$ & $\begin{array}{c}-2.654 * * \\
(1.042)\end{array}$ \\
\hline $\ln ($ alpha) & $\begin{array}{l}-14.618^{*} \\
(7.488)\end{array}$ & $\begin{array}{l}-0.512 * * \\
(0.202)\end{array}$ & $\begin{array}{l}1.345^{* * *} \\
(0.36)\end{array}$ & $\begin{array}{l}0.84 * * \\
(0.381)\end{array}$ \\
\hline
\end{tabular}

Nguồn: Kết quả phân tích dữ liệu của nhóm nghiên cứu

Hệ số ước lượng của nhóm biến giả trình độ học vấn cho thấy trình độ học vấn ch làm thay đổi tần suất mua rau tại cửa hàng RAT chứ không tác động đến các kênh khác. Nhìn chung người có trình độ cao hơn sẽ có xu hướng mua rau tại cửa hàng RAT nhiều hơn. Tuy nhiên, người có trình độ sau đại học lại ít mua rau ở cửa hàng RAT và chợ hơn. Nhóm người này nhìn chung ít mua rau hơn các nhóm khác.

Người mua thuộc nhóm nghề nghiệp khác nhau nhìn chung không khác biệt nhau trong tần suất mua rau tại siêu thị, cửa hàng RAT và các kênh khác. Tuy nhiên có thể nhận thấy rằng nhóm nhân viên văn phòng và công nhân mua rau ở chợ truyền thống nhiều hơn hẳn so với nhóm quản lý. Công nhân cũng mua rau ở cửa hàng RAT ít hơn đáng kể so với nhóm quản lý. Ngoài ra kết quả còn cho thấy nhóm quản lý không khác biệt so với nhóm nội trợ xét về tần suất mua rau giữa các kênh.

Hai biến số về lòng tin đối với người bán và quản lý nhà nước có tác động rất rõ. Những người tin vào người bán, hoặc không tin vào sự quản lý của chính phủ về VSATTP ít mua rau ở chợ truyền thống hơn hẳn, đồng thời mua rau ở siêu thị nhiều hơn hẳn. Kết quả này xác nhận một phần giả thuyết $\mathrm{H} 1$. Tuy nhiên tác động của lòng tin đến tần suất mua rau ở cửa hàng và các kênh khác là không rõ ràng.

Các kênh thông tin có tác động khác nhau đến tần suất mua rau ở các kênh bán rau. Nhóm tìm hiểu thông tin qua truyền thanh truyền hình mua rau ở siêu thị nhiều hơn nhóm không tìm hiểu thông tin qua kênh này. Tuy nhiên kênh thông tin này lại không ảnh hưởng đến tần suất mua rau tại chợ, cửa hàng RAT và các kênh khác.

Thông tin từ internet hầu như không có tác động, ngoại trừ việc làm giảm tần suất mua rau tại các cửa hàng RAT. Báo chí lại có tác động tương tự như truyền thanh truyền hình: làm tăng số lần mua rau tại siêu thị trong khi không ảnh hưởng đến tần suất mua rau tại các kênh khác. Riêng những người tìm hiểu thông tin qua kênh truyền miệng ít mua rau ở chợ hơn hẳn và chuyển qua mua rau tại các cửa hàng RAT. Nhìn chung ch có thông tin truyền thanh truyền hình và báo chí là xác nhận một phần giả thuyết $\mathrm{H} 2$ : làm tăng tần suất mua rau ở siêu thị. Tuy 
nhiên ngay cả hai nguồn tin này cũng không làm giảm tần suất mua rau ở chợ truyền thống và cũng không làm tăng tần suất mua ở cửa hàng rau an toàn. Các kênh thông tin còn lại đều bác bỏ $\mathrm{H} 2$.

\section{Kết luận}

Nghiên cứu phân tích tác động của lòng tin và kênh thông tin VSATTP đến tần suất mua rau tại chợ truyền thống, siêu thị, cửa hàng RAT và các kênh khác. Số liệu thu thập từ 254 người mua rau tại bốn quận huyện ở TP.HCM. Mô hình SUNB được áp dụng để ước lượng 4 phương trình đồng thời nhằm giải thích tần suất mua rau mỗi tuần tại các kênh bán lẻ.

Kết quả nghiên cứu cho thấy những người tin tưởng người bán rau có xu hướng mua ở siêu thị nhiều hơn và ít mua ở chợ. Những người cho rằng quản lý nhà nước về VSATTP là yếu kém cũng có xu hướng mua rau ở siêu thị nhiều hơn và ít mua ở chợ hơn. Điều này cho thấy việc cải thiện lòng tin của người dân về vai trò quản lý VSATTP của chính phủ sẽ làm tăng tần suất mua rau ở chợ.

Kết quả hồi quy mô hình SUNB cũng cho thấy các kênh thông tin có tác động khác nhau đến tần suất mua rau ở mỗi kênh bán lẻ. Kênh thông tin duy nhất có khả năng ảnh hưởng đến tần suất mua rau ở chợ là truyền miệng. Những người tìm hiểu thông tin về VSATTP qua kênh này ít mua rau ở chợ hơn hẳn. Tuy nhiên kênh thông tin này lại không tác động đến tần suất mua rau tại các kênh bán lẻ khác. Có lẽ là do những lời truyền miệng chủ yếu là nói xấu về tình trạng mất vệ sinh ở chợ truyền thống.

Có thể làm tăng tần suất mua rau ở siêu thị thông qua kênh truyền thanh truyền hình và báo chí vì những người tìm hiểu thông tin qua hai kênh này có tần suất mua rau ở siêu thị cao hơn hẳn. Tuy nhiên hai kênh thông tin này lại không có tác động đến tần suất mua rau tại các kênh bán lẻ khác.

Cửa hàng RAT có thể nhờ kênh truyền miệng để tăng lượng khách hàng vì những người tìm hiểu thông tin qua kênh truyền miệng có xu hướng mua rau ở cửa hàng RAT nhiều hơn. Tuy nhiên kênh thông tin qua internet lại đang làm giảm tần suất mua rau tại các cửa hàng RAT. Cuối cùng, các kênh bán lẻ khác không bị ảnh hưởng đáng kể bởi các kênh thông tin.

Nghiên cứu này cũng có một số hạn chế. Một là mô hình hồi quy có thể thiếu một vài biến quan trọng, ví dụ giá hoặc chênh lệch giá giữa các kênh. Tuy nhiên việc đưa biến giá của các kênh vào mô hình dạng này hơi phức tạp và có lẽ nên dùng Multivariate Negative Binomial sẽ phù hợp hơn. Một hạn chế nữa có thể là vấn đề nội sinh có thể xảy ra ở một số biến, ví dụ lòng tin và kênh tìm hiểu thông tin. Ví dụ người tin ở siêu thị sẽ có xu hướng mua ở siêu thị nhiều hơn, kết quả là có thể có mối quan hệ nhân quả ngược ở biến lòng tin đối với người bán. Cuối cùng, cỡ mẫu của nghiên cứu này còn hạn chế và chưa được thu thập ở tất cả các địa bàn của TP.HCM và do vậy có thể chưa thật sự đại diện cho sở thích của người dân TP.HCM. 


\section{Tài liệu tham khảo}

Bai, J., Wahl, T. I., \& McCluskey, J. J. (2008). Consumer choice of retail food store formats in Qingdao, China. Journal of International Food \& Agribusiness Marketing, 20(2), 89-109.

Bond, J. K., Thilmany, D., \& Bond, C. (2009). What influences consumer choice of fresh produce purchase location? Journal of Agricultural and Applied Economics, 41(1), 6174.

Cadilhon, J. J., Moustier, P., Poole, N. D., Tam, P. T., \& Fearne, A. P. (2006). Traditional vs. modern food systems? Insights from vegetable supply chains to Ho Chi Minh City. Development Policy Review, 24(1), 31-49.

Carpenter, J., \& Moore, M. (2006). Consumer demographics, store attributes, and retail format choice in the US grocery market. International Journal of Retail \& Distribution Management, 34(6), 434-452.

Chen, S. L., Chern, W. S., Lin, Y.-R., \& Liu, K. E. (2015). Effects offood safety and health risk information on demand for food in Taiwan. Paper presented at Agricultural \& Applied Economics Association and Western Agricultural Economics Association Annual Meeting, San Francisco, CA.

Cheng, L., Jiang, S., Zhang, S., You, H., Zhang, J., \& Xiao, Y. (2016). Consumers' behaviors and concerns on fresh vegetable purchase and safety in Beijing urban areas, China. Food Control, 63, 101-109.

Dong, D., \& Stewart, H. (2012). Modeling a household's choice among food store types. American Journal of Agricultural Economics, 94(3), 702-717.

Gido, E. O., Ayuya, O. I., Owuor, G., \& Bokelmann, W. (2016). Consumer's choice of retail outlets for African indigenous vegetables: Empirical evidence among rural and urban households in Kenya. Cogent Food \& Agriculture, 2, 1-14.

Goldman, A., \& Hino, H. (2005). Suppermarkets vs. traditional retail stores: Diagnosing the barriers to suppermarkets' market share growth in an ethnic minority community. Journal of Retailing and Consumer Services, 12, 273-284.

Gorton, M., Sauer, J., \& Supatpongkul, P. (2011). Wet market, supermarkets and the "Big Middle" for food retailing in developing countries: Evidence from Thailand. World Development, 39(9), 1624-1637.

Hoang, H., \& Nakayasu, A. (2006). Study on the factors influencing the consumption of safe vegetables in Ho Chi Minh City, Vietnam. Journal of Applied Sciences, 6, 1986-1992.

Huang, Z., Pan, X.-D., Wu, P.-G., Han, J.-L., \& Chen, Q. (2014). Heavy metals in vegetables and the health risk to population in Zhejiang, China. Food Control, 36, 248-252.

Iton, C. A. (2015). Factors influencing retail outlet choice of women purchasing fresh fruits in Trinidad and Tobago. American Journal of Business and Management, 4(1), 38-48. 
Kyureghian, G., \& Nayga, R. (2013). Food store access, availability, and choice when purchasing fruits and vegetables. American Journal of Agricultural Economics, 95(5), 1280-1286.

Lapar, M. L., Toan, N. N., Que, N. N., Jabbar, M., Tisdell, C., \& Staal, S. (2009). Market outlet choices in the context of changing demand for fresh meat: Implications for smallholder inclusion in pork supply chain in Vietnam. Paper presented at the International Association of Agricultural Economists Conference, China.

Maples, M., Morgan, K., Interis, M., \& Harri, A. (2013). Who buys food directly from producers in the Southeastern United States? Journal of Agricultural and Applied Economics, 45(3), 509-518.

Maruyama, M., \& Trung, L. V. (2007). Traditional bazaar or supermarkets: A probit analysis of affluent consumer perceptions in Hanoi. The International Review of Retail, Distribution and Consumer Research, 17(3), 233-252.

Maruyaman, M., \& Wu, L. (2014). Quantifying barriers impeding the diffusion of supermarkets in China: The role of shopping habits. Journal of Retailing and Consumer Services, 21, 383-393.

Maruyama, M., Wu, L., \& Huang, L. (2016). The modernization of fresh food retailing in China: The role of customers. Journal of Retailing and Consumer Services, 30, 33-39.

Melis, K., Campo, K., Breugelmans, E., \& Lamey, L. (2015). The impact of the multi-channel retail mix on online store choice: Does online experience matter? Journal of Retailing, 91(2), 272-288.

Meng, T., Florkowski, W. J., Sarpong, D. B., Chinnan, M. S., \& Resurreccion, A. V. (2014). Consumer's food shopping choice in Ghana: Supermarket or traditional outlets? International Food and Agribusiness Management Review, 17, 107-130.

Mergenthaler, M., Weinberger, K., \& Qaim, M. (2009). The food system transformation in developing countries: A disaggregate demand analysis for fruits and vegetables in Vietnam. Food Policy, 34(5), 426-436.

Mo, L. (2013). Impact of food safety information on US poultry demand. Applied Economics, $45,1121-1131$.

Neven, D., Reardon, T., Chege, J., \& Wang, H. (2006). Supermarkets and consumers in Africa. Journal of International Food \& Agribusiness Marketing, 18(2), 103-123.

Okello, J., Lagerkvist, C.-J., Hess, S., Ngigi, M., \& Karanja, N. (2011). Choice of fresh vegetable retail outlets by developing-country urban consumers: The Case of Kale consumers in Nairobi, Kenya. European Journal of Development Research, 24, 434-449.

Palma, M., Emerson, R., \& House, L. (2003). Evaluating factors influencing grocery store choice. Paper presented at the American Agricultural Economics Association Annual Meeting, Montreal, Canada. 
Paudel, L., Adhikari, M., \& Houston, J. E. (2005). Assessing the impacts of low carbohydrate related health information on the market demand for vegetables. Paper presented at the American Agricultural Economics Association Annual Meeting, Rhode Island.

Reardon, T. (2006). The rapid rise of supermarkets and the use of private standards in their food product procurement systems in developing countries. Agro-food Chains and Networks for Development, 79-105.

Reardon, T., Timmer, P., Barrett, C., \& Berdegué, J. (2003). The rise of supermarkets in Africa, Asia, and Latin America. American Journal of Agricultural Economics, 85(5), 11401146.

Slamet, A. S., \& Nakayasu, A. (2016). Consumers' choice for vegetable market channels in Indonesia. ICoA Conference Proceedings, 3, 167-172.

Smed, S., \& Jensen, J. D. (2005). Food safety information and food demand. British Food Journal, 107(3), 173-186.

Sở NN\&PTNN. (2016). Báo cáo kết quả điều tra thị hiếu người tiêu dùng về sản phẩm VietGAP [Report the results of surveying consumer tastes about VietGAP products]. Retrieved June 15, 2017, from http://www.sonongnghiep.hochiminhcity.gov.vn/default.aspx

Staus, A. (2011). Which household attitudes determine the store type choice for meat? Journal of Retailing and Consumer Services, 18, 224-234.

VEBIMO. (2010). Kinh tế nông nghiệp Việt Nam. Nghiên cứu phân tích kinh tế Vebimo, 1, 128.

Verhoef, P. C., Neslin, S. A., \& Vroomen, B. (2007). Multichannel customer management: Understanding the research-shopper phenomenon. International Journal of Research in Marketing, 24, 129-148.

Wertheim-Heck, S. C. (2015). We have to eat, right? Food safety concerns and shopping for daily vegetables in modernizing Vietnam (Doctoral dissertation). Wageningen University, Wageningen, Netherlands.

Winkelmann, R. (2000). Seemingly unrelated negative binomial regression. Oxford Bulletin of Economics and Statistic, 62(4), 553-560.

Zepeda, L. (2009). Which little piggy goes to market? Characteristics of US farmers' market shoppers. International Journal of Consumer Studies, 33, 250-257.

Zepeda, L., \& Li, J. (2006). Who buys local food? Journal of Food Distribution Research, $37(3), 1-11$. 